\title{
Global dermatology: challenges and changes?
}

\author{
G. Tchernev · J. W. Patterson - A. A. Chokoeva
}

Published online: 24 August 2016

(C) Springer-Verlag Wien 2016

The achievements of contemporary medical science, diagnostics and therapy as well as the global experience gained in recent decades have led to an entirely new era in the understanding and practice of medicine in all its fields worldwide.

Despite this, the therapy of numerous immunological disorders still remains a challenge, in view of the often unsatisfactory therapeutic responses in this category of diseases. Although various new hypotheses have been reported recently, leading to the discovery of unexpected information regarding the pathogenesis of some diseases, such as Pyoderma gangrenosum, the lack of a unified treatment regimen with a certain positive therapeutic outcome emphasizes that there may still be undiscovered components in the pathogenetic chain. It is possible that refractoriness to the use of biological agents as monotherapy stems from the fact that a single agent cannot affect the entire inflammatory cascade or influence simultaneously all of its stages [1]. The specific therapy should aim to attack more than one link in the inflammatory cascade

Prof. G. Tchernev $(\bowtie)$

Department of Dermatology, Venereology and Dermatologic Surgery, Medical Institute of Ministry of Interior (MVR), General Skobelev blvd. 79, 1606 Sofia, Bulgaria georgi_tchernev@yahoo.de

J. W. Patterson, MD

Department of Pathology, University of Virginia Health System, 1215 Lee Street, Box 800214, Charlottesville, VA 22908, USA

jwp9e@virginia.edu

Dr. A. A. Chokoeva

Onkoderma-Policlinic for Dermatology and Dermatologic Surgery, General Skobelev 26 blvd., Sofia, Bulgaria assia_chokoeva@abv.bg

Department of Dermatology and Venereology, Medical University of Plovdiv, Medical Faculty, Plovdiv, Bulgaria in order to achieve the maximum therapeutic effectiveness [1]. We believe that this could be achieved with combined therapy with different groups of biological agents (for example, combined therapy with an IL-1 receptor antagonist and a tumor necrosis factor$\alpha$ inhibitor [TNF- $\alpha$ inhibitor]) [1].

Interesting new discoveries have also been reported regarding certain infectious diseases. Recent data show increasingly changing patterns in the epidemiology of the most common dermatophytes that cause tinea capitis [2]. The former leading causes of this disorder, such as Trichophyton schoenleinii, are nowadays less commonly cultured, while other species previously regarded as "nonpathogenic" may be seen as the main causative agent in some atypical forms of the disease [2]. Molds should be considered as a potential source of infection in cases of resistant or atypical tinea capitis rather than simply accompaniments of the disease, particularly in some geographic regions. The reorganization of etiologies requires reconsideration of former therapeutic concepts, in view of the higher antibiotic resistance of molds when compared to dermatophytes [2].

Some aspects in the field of dermatologic oncology also bring us "back to the future," despite the recent progress in genetics and targeted therapy. As an example, the recommended management for dysplastic nevi in the framework of the so-called atypical mole syndrome was focused on the screening and regular observation with dermatoscopy or confocal microscopy. However, the changing patterns of oncogenesis, leading to malignant transformation, cannot always be detected in early stages and, as a result, the diagnosis of melanoma is often delayed. Subsequently, the old mode of early surgical eradication of every suspicious lesion is much more effective in these patients, particularly for patients with a family history of melanoma and especially their children. 
The heterogeneous nature and genetics of malignant melanoma make its behavior unpredictable, and for that reason, we postulate that an early, aggressive surgical approach would be of greatest benefit in many cases.

Conflict of interest G. Tchernev, J.W. Patterson and A.A. Chokoeva declare that they have no competing interests.

\section{References}

1. Chokoeva AA, Cardoso JC, Wollina U, Tchernev G. Pyoderma gangrenosum-a novel approach? Wien Med Wochenschr. 2016. doi:10.1007/s10354-016-0472-z.

2. Chokoeva AA, Zisova L, Chorleva K, Tchernev G. Aspergillus niger - a possible new etiopathogenic agent in Tinea capitis? Presentation of two cases. Braz J Infect Dis. 2016;20(3):303-7. 